\title{
Artículos
}

\section{Métodos cuantitativos de evaluación de la ciencia: bibliometría, cienciometría \\ e informetría ${ }^{1}$}

\author{
NADIA VANTI \\ Instituto Latino-Americano de Estudios Avanzados de la Universidade Federal \\ do Rio Grande do Sul (UFRGS), Cristiano Fischer, 2320/ 202 91410-000 \\ Porto Alegre - RS/ Brasil, Tel. ++ 5551 336-3737 \\ E-mail: nvanti@ilea.ufrgs.brq.
}

\section{Trabajo recibido el \\ 12 de octubre de $\mathbf{2 0 0 0}$$$
\text { * }
$$$$
\text { Trabajo }
$$$$
\text { aceptado el } 15
$$ de diciembre de 2000}

\section{RESUMEN}

Presenta las diversas definiciones sobre bibliometría, cienciometría e informetría encontradas en la literatura y revela la difícil delimitación que aún existe entre estos distintos subcampos. A partir de esta conceptualización se pretende mostrar la aplicabilidad de cada uno de estos métodos cuantitativos y su importancia como subsidio para evaluar las diferentes áreas del conocimiento científico, incluyendo las Ciencias de la Información.

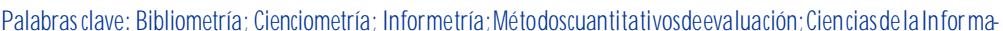 dớn}

\section{QUANTITATIVE ASSESSMENT METHODS OF SCIENCE: BIBLIO-METRICS, INFO-METRICS AND SCIENTIFICMEASUREMENT \\ NADIA VANTI}

1 Agradezco los valiosos comentarios y sugerencias de mi orientador Silas Marques de Oliveira, y de Else Benetti Marques Valio. 


\section{INTRODUCCIÓN}

Ta idea de evaluar la ciencia surgió en este siglo cuando, después de la Primera LGuerra Mundial, se percibió que a pesar de sus grandes progresos no había sido aún capaz de solucionar problemas sociales importantes como la mala distribución de los ingresos, la subalimentación y las diversas enfermedades que afectaban a vastos sectores de la población mundial, mientras que por otro lado estaba siendo capaz de producir armas de guerra cada vez más poderosas. La ciencia debía dar una satisfacción a la sociedad. Con esa finalidad los países desarrollados comenzaron a adoptar técnicas e instrumentos más explícitos que permitieran la detección y la comprensión de las actividades científicas (Velho, 1985).

Con el correr del tiempo la evaluación se fue convirtiendo en un procedimiento cada vez más utilizado como ayuda para hacer la selección necesaria ante la creciente producción del mundo científico y la consecuente escasez de recursos para financiar todas las propuestas, grupos de investigación, instituciones y cursos que estaban floreciendo. Principalmente en los países del Primer Mundo, donde la comunidad académica pasó a ser muy activa, se exigió por parte de los órganos de financiación un sistema de valoración eficaz que pudiera liberar recursos para aquellos proyectos que representasen realmente una contribución significativa para la ciencia. Más precisamente en los años 60, cuando los recursos para ciencia y tecnología comenzaron a reducirse, ese control se tornó más frecuente (Castro, 1985).

La necesidad de evaluar la ciencia a causa del elevado volumen de producción científica en el mundo actual es confirmada por Braga (1974, p. 157) cuando afirma que:

De un modo general, el número de científicos y documentos científicos se decuplica en el mismo período en que se duplica la población mundial. La ciencia es el producto de crecimiento más rápido de nuestra civilización y, durante más de tres siglos ha venido explotando con increíble impacto[... $]^{2}$

Los indicadores científicos son cada vez más necesarios para aquellos que formulan las políticas científicas en el plano nacional, quienes deben determinar las prioridades de investigación entre los más diversos campos científicos y aun dentro de ellos. Los responsables de la toma de decisiones, tanto en los órganos de gobierno como en las entidades de financiamiento, necesitan contar con datos sistemáticos sobre el desempeño de las distintas áreas a fin de poder escoger, con mejores fundamentos, dónde concentrar los limitados recursos financieros y humanos de que disponen (Irvine \& Martin, 1989).

La evaluación más sistemática y la formalización de sus procedimientos tuvo origen en elOfficeforNaval Reserchde Estados Unidos, una institución pública que pasó a financiar proyectos e investigaciones científicas. Existen actualmente dos criterios de evaluación en el campo de la investigación científica: uno de ellos, el más tradicio-

2 Ésta y las siguientes citas de textos que no estaban en castellano fueron traducidas 
nalmente utilizado, es el de la evaluación hecha por los pares, en la que el statusdel investigador tiene gran influencia en la decisión. Por tratarse de un criterio excesivamente subjetivo y, por lo tanto difícil de padronizar o modelizar, se ha empezado a recurrir con mayor frecuencia al uso de métodos cuantitativos, ya sea para sustituir ese primer criterio o para complementarlo. Es en este sentido que se comienzan a aplicar las técnicas bibliométricas, cienciométricas e informétricas, como forma de tornar más objetivos, y consecuentemente más confiables, los criterios de evaluación (Castro, 1985). Sin embargo para alcanzar un mayor equilibrio entre la subjetividad y la objetividad en la evaluación de la ciencia, tanto Castro (1985) como Sandstrom \& Sandstrom (1995) y Bowen (1999) recomiendan la combinación de ambos criterios a fin de reforzar su validación. El desarrollo de técnicas cuantitativas para apreciar las ciencias viene a contribuir, efectivamente, a lograr una evaluación más imparcial, completa y rigurosa que la que puede ser obtenida mediante la utilización de métodos puramente cualitativos.

Tratando de definir las dos áreas que tienen como objetivo la medición del conocimiento, Castro (1985, p. 30) afirma que:

[...]la bibliometría y la cienciometría no son más que técnicas para tratar comparaciones de forma más completa y rigurosa. Sus ingredientes básicos son, pues, el reaprovechamiento de evaluaciones generadas para otros fines y las técnicas cuantitativas para su tratamiento.

Según este autor las ventajas de la bibliometría radican en que facilitan los elementos de juicio y producen resultados cuantitativos que tienden a ser la suma de muchos pequeños juicios y apreciaciones realizadas por varias personas. Dado que una gran parte de la producción científica se torna conocida mediante su publicación, es a través de ésta como resulta más fácil la evaluación de las actividades de investigación.

Para la mayoría de los que actúan en la investigación la publicación representa no sólo un indicador sino el producto final de todo un esfuerzo creativo. De esa forma cualquier contribución sólo es reconocida luego de ser publicada, juzgada e incorporada, de alguna manera, a los conocimientos ya existentes. El ciclo del conocimiento sólo se completa con la aceptación del descubrimiento por parte de otros científicos de la misma área, y eso se da a través de la publicación. Por ese motivo es importante evaluar un área de conocimiento siguiendo los pasos de la publicación científica de uno o varios investigadores de determinado campo de la ciencia (Braga, 1974).

Sin embargo, además de la publicación existen otros indicadores que pueden ser considerados al momento de hacer una evaluación científica. De acuerdo con Velho $(1985$, p. 36),

[...]se puede decir que se han usado cuatro tipos principales de medidas para construir indicadores científicos: medidas de input tanto desde el punto de vista de los recursos financieros como del personal involucrado en la actividad (número de científicos); recuento del número de premios honoríficos recibidos por los miembros de determinada 
comunidad; recuento del número de publicaciones y recuento del número de citas hechas a los artículos publicados.

Por otro lado, Moravcsik (cit. por Velho, 1985, p. 36), sugiere que "existen tres aspectos de la ciencia que pueden ser medidos: actividad, productividad y progreso". Y los define así:

[...] sintéticamente, actividad significa algo como el consumo relevante de los recursos de imput Productividad significa el grado en que tal consumo produce resultados relevantes. Finalmente, progreso también mide el grado en que esa productividad nos acerca a alcanzar ciertos objetivos específicos.

Velho (1985, p. 36), analizando el pensamiento de Moravcsik, concluye que [...] el progreso puede ser entendido, de cierta forma, como una medida de la calidad de toda contribución hecha al conocimiento científico. Implícitamente, la mayoría de los analistas de esa área asume que medidas de inputindican actividad, recuento de premios honoríficos y citas recibidas por un artículo científico indican progreso o calidad.

El Institutefor SäentificInfomation(ISI), organización fundada por Eugene Garfield en Filadelfia, Estados Unidos, se ha constituido en una importante fuente de datos para muchas de estas mediciones. Esa organización procesa anualmente un alto número de periódicos que cuentan con artículos que abarcan más de 100 sub-áreas altamente especializadas del conocimiento científico. En sus comienzos las informaciones publicadas por este instituto parecen haber sido más utilizadas por los historiadores y sociólogos de la ciencia para explorar aspectos como la ramificación de la ciencia y el crecimiento del conocimiento científico. A partir de la década del 80, luego de que este instituto vendiera su base de datos a diferentes instituciones como herramienta auxiliar en la elaboración de la política científica, la säentomenics (cuantificación de la ciencia) comienza a ser un área de interés académico. Actualmente esta disciplina está siendo ampliamente utilizada para medir el conocimiento científico. En este sentido tiene una gran variedad de aplicaciones, como por ejemplo en la utilización del número de publicaciones y citas como auxiliar en la evaluación del desempeño científico de los investigadores, grupos y centros de investigación; en la toma de decisiones en cuanto a la distribución de recursos financieros por parte de organizaciones gubernamentales, fundaciones e instituciones de fomento de determinadas áreas de conocimiento; en el análisis de citas para prever áreas emergentes de interés tecnológico dentro de la ciencia y, en fin, en el estudio comparativo del desempeño de ciertas naciones (Velho, 1985).

El ISI cubre, a través del SaieneCitationIndex (SCI), la mayoría de las revistas internacionales importantes en el área de las ciencias puras, aplicadas y médicas. Por su parte el Social SáenceCitationIndex (SSCI) cumple la misma función para el área de las ciencias sociales (Rousseau, 1998), y el Arts \& Humanities Citation Index (A\&HCI) cumple el mismo papel para las áreas de artes y humanidades (ISI, 2000). 


\section{BIBLIOMETRÍA, CIENCIOMETRÍA E INFORMETRÍA}

La palabra evaluar viene del latín valee Ésta presenta, entre otras acepciones, la de ser merecedor o digno de alguna cosa (cf Torrinha, 1939; Heckler \&al., 1984; Alves, 1999). La evaluación, dentro de una determinada rama del conocimiento, permite dignificar este saber siempre que se utilicen métodos sistemáticos y confiables para demostrarle a la sociedad de qué manera se viene desenvolviendo y de qué forma ha contribuido para resolver los problemas que se presentan dentro de su área de incumbencia.

Existe por parte de autores como Oliveira \&al. (1992, p. 239), por ejemplo, la idea de que

[...]la evaluación de la productividad científica debe ser uno de los elementos principales para el establecimiento y acompañamiento de una política nacional de enseñanza e investigación, ya que permite un diagnóstico de las reales potencialidades de los grupos y/o instituiciones, académicas o no.

Se cuestiona, sin embargo, de qué manera es posible hacer este diagnóstico. Una de las posibilidades consiste en utilizar métodos que permitan medir la productividad de los investigadores, grupos o instituciones de investigación. Para ello se requiere el uso de técnicas específicas de evaluación que pueden ser cuantitativas o cualitativas o una combinación de ambas. Las técnicas cuantitativas de evaluación

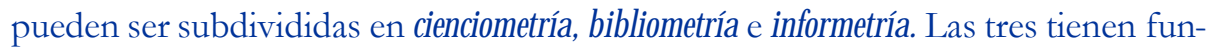
ciones semejantes, pero, al mismo tiempo cada una de ellas propone medir la ciencia bajo enfoques diferentes. Existen aún dificultades para establecer dónde termina una y comienza la otra. Para aclarar mejor las semejanzas y diferencias entre estas técnicas se buscó en la literatura del área, además de hacer un breve historial de la utilización de los tres términos con base en algunas definiciones elaboradas por investigadores del tema.

Aunque según autores como Lawani (1981) y Sengupta (1992) el término bibliometría habría sido acuñado por Alan Pritchard en 1969, Fonseca (1973) ha demostrado que quien realmente utilizó por primera vez este término fue Paul Otlet varias décadas antes en su obra titulada Traitédedoumetation de 1934. En verdad Pritchard (1969) popularizó el uso de la palabra "bibliometría" cuando sugirió que ésta debería sustituir al término "bibliografía estadística", que venía siendo utilizado desde la mención hecha en 1922 por Edward Wyndham Hulme en una conferencia en la Universidad de Cambridge en la que se remitiera a un estudio pionero de Cole yEales, de 1917, referido al análisis estadístico de una bibliografía de Anatomía Comparada (véase también Fonseca, 1986). De cualquier forma, sería importante no olvidar que, tal como señala Shapiro (1992), las técnicas bibliométricas - más allá de la cuestión del origen y uso del término - ya eran aplicadas bastante antes que en el estudio de Coles y Eales, en el campo del Derecho, donde los análisis de citas legales y la indexación de jurisprudencia datan de épocas mucho más remotas. 
En 1948, en la Conferencia de la Aslib en Leamington Spa, Ranganathan sugería la necesidad de que los bibliotecarios desarrollaran la "bibliotecometría", ya que las bibliotecas lidiaban con una gran cantidad de números. Sin embargo, fue hasta 1969, en el seminario anual del Doumentation ReserchandTrainingCentre(DRTC), cuando presentó un trabajo con algunos ejemplos de aplicación de la estadística a la biblioteconomía. Fue luego de basarse en este trabajo de Ranganathan y en investigaciones realizadas en el DRTC, cuando Neelameghan (1969) esbozó la aplicabilidad de la bibliotecometría, o bibliometría, como pasó a ser conocida (Ravichandra Rao, 1986).

El término cienciometría surgió en la URSS y Europa Oriental y fue empleado especialmente en Hungría. Entre los primeros autores que lo utilizaron están Dobrov y Karennoi (1969) en una publicación del All-UnionInstitutfor SáentificandTechnical Information(VINITI). Originalmente se refería a la aplicación de métodos cuantitativos para el estudio de la historia de la ciencia y del progreso tecnológico (Egghe cit. por Spinak, 1996). Las primeras definiciones consideraban a la cienciometría como "la medición del proceso informático", donde el término "informático" significaba, a diferencia de hoy, "la disciplina científica que estudia la estructura y las propiedades de la información científica y las leyes del proceso de comunicación" (Mikhilov đal. cit. por Spinak, 1996). Este término alcanzó notoriedad con el inicio de la publicación, en 1977, de la revista Säentamerics editada originalmente en Hungría y actualmente en Holanda (Tague-Sutckiffe, 1992).

De acuerdo con Brookes (1990), el término informetría fue propuesto por primera vez por Otto Nacke, director del Institutfür Informtrię en Bielferd, Alemania, en 1979. El término fue adoptado inmediatamente por el VINITI en la antigua URSS, institución que impulsó la creación de un comité con ese nombre en la Federación Internacional de Documentación: el FID/ IM-Committeen Infometry, indicando al propio Nacke como su primer director. Su aceptación definitiva data de 1989, cuando el Encuentro Internacional de Bibliometría pasó a llamarse Conferenaia Intemadio nal deBibliomđńa, CieniomeńaeInfomđnáasiguiendo la sugerencia de Brookes, hecha dos años antes en la Conferencia de Diepenbeek, Bélgica (Tague-Sutckiffe, 1992).

Sengputa (1992) y Gläzel \& Schoepflin (1994), por ejemplo, presentan los tres términos como sinónimos, mientras que Gorkova (cit. por Brookes 1990), Russell (1994), Egghe (1994) y Tague-Sutckiffe (1992), entre otros, ya consideran que la informetría comprende un campo más amplio que la bibliometría y que, incluso, engloba también a la cienciometría.

De acuerdo con las palabras de este último autor, traducidas por Macias-Chapula (1998, p. 134), se puede definir la bibliometría como:

[...]el estudio de los aspectos cuantitativos de la producción, difusión y uso de la información registrada. La bibliometría desarrolla patrones y modelos matemáticos para medir esos procesos, usando sus resultados para elaborar previsiones y apoyar tomas de decisiones.

Tague-Sutckiffe (1992), hablando sobre la cienciometría, y siguiendo con la traducción de Macias-Chapula (1998, p.134), la define en los siguientes términos: 
Cienciometría es el estudio de los aspectos cuantitativos de la ciencia en tanto que disciplina o actividad económica. La cienciometría es un segmento de la sociología de la ciencia, que es aplicada en el desarrollo de políticas científicas. Abarca estudios cuantitativos de las actividades científicas, incluyendo la publicación y superponiéndose, por lo tanto, a la bibliometría.

Según la definición de Tague-Sutckiffe, la cienciometría estudia, por medio de indicadores cuantitativos, una determinada disciplina de la ciencia. Estos indicadores cuantitativos son utilizados dentro de un área del conocimiento, por ejemplo a través del análisis de publicaciones, y se aplican en el desarrollo de políticas científicas. La cienciometría intenta medir los incrementos de producción y productividad de una disciplina o de un grupo de investigadores de un área para delinear el crecimiento de determinada rama del conocimiento.

Para Van Raan (1997), la cienciometría se dedica a realizar estudios cuantitativos en ciencia y tecnología y a descubrir los lazos existentes entre ambas, apuntando al avance del conocimiento y tratando de relacionar éste con cuestiones sociales y de políticas públicas. La cienciometría tendría, por lo tanto, un carácter multidisciplinario en lo que se refiere a los métodos que utiliza. Tales métodos provienen tanto de las ciencias naturales como de las ciencias sociales y del comportamiento (estadística y otros métodos matemáticos, modelos sociológicos, investigaciones y métodos psicológicos de entrevista, informática, filosofía de la ciencia, lingüística, etcétera).

Analizando la definición de Tague-Sutckiffe sobre bibliometría e intentando compararla con su conceptualización de cienciometría, se nota que existe una sutil diferencia entre ambas. La bibliometría estaría más dirigida a un análisis cuantitativo de determinados procesos de información, incluyendo la publicación pero extendiéndose a la distribución y el uso de la información. La finalidad de la bibliometría, así como la de la cienciometría, sería utilizar los resultados de las mediciones para la previsión y la toma de decisiones. Llama la atención, sin embargo, que en ningún momento el autor establezca una relación entre los procesos de información y una disciplina o ciencia específica. Se tiene la impresión de que la medición, en la bibliometría, estaría dirigida al proceso y no especialmente a una determinada rama de la ciencia como en el caso de la cienciometría.

Spinak (1998, p. 142) confirma la interdisciplinariedad de la bibliometría y su enfoque hacia las fuentes de información cuando presenta su definición: "Labibliome tńa puss, unadisciplinaconalcancemultidisaiplinanioyla queanalizaunodelosaspeetosmás redeantesy djeivos desa commidad, la comnidadimpresa”.

Tratando de hacer una distinción entre bibliometría y cienciometría, ya Spinak (1998, p. 143) afirma también que:

La bibliometría estudia la organización de los sectores científicos y tecnológicos a partir de las fuentes bibliográficas y patentes para identificar los actores, sus relaciones y sus tendencias. Por el contrario, la cienciometría trata con las varias mediciones de la literatura, de los documentos 
y otros medios de comunicación, mientras que la bibliometría tiene que ver con la productividad y utilidad científica.

El mismo Spinak (1998, p. 142), haciendo una relación entre bibliometría y cienciometría, afirma:

La cienciometría aplica técnicas bibliométricas a la ciencia ... pero va mas allá de las técnicas bibliométricas, pues también examina el desarollo y las políticas científicas. [...] la cienciometría puede establecer comparaciones entre las políticas de investigación entre los países analizando sus aspectos económicos y sociales.

Por último, refiriéndose a la informetría, la más nueva de las tres técnicas cuantitativas analizadas, se puede decir que ésta tiene un propósito más claro y abarcador que la cienciometría y la bibliometría pues, siempre de acuerdo con la conceptualización de Tangue-Sutckiffe (1992) glosada por Macias-Chapula (1998, p. 134), Informetría es el estudio de los aspectos cuantitativos de la información en cualquier formato y no sólo registros catalográficos o bibliografías, referido a cualquier grupo social y no sólo a los científicos. La informetría puede incorporar, utilizar y ampliar los muchos estudios de evaluación de la información que se hallan fuera de los límites de la bibliometría y la cienciometría:

La informetría se distinguiría claramente de la cienciometría y de la bibliometría en lo que se refiere al universo de objetos y sujetos que estudia, ya que no se limita sólo a la información registrada, sino que puede analizar también los procesos de comunicación informal, incluso hablada, y dedicarse a investigar los usos y necesidades de información de los grupos sociales desfavorecidos y no sólo los de las elites intelectuales (Tague-Sutckiffe, 1992).

Según Wormell (1998, p. 2), “[...]la informetría es un sub-campo emergente de la Ciencia de la Información, y está basada en la combinación de técnicas avanzadas de recuperación de la información con estudios cuantitativos de los flujos de información”. Para Wolfram (1992a), la informetría encuentra su utilidad en la administración de colecciones en bibliotecas, en el desarrollo de políticas científicas, y puede ayudar en la toma de decisiones en relación al diseño y mantenimiento de sistemas de recuperación de información.

Las definiciones teóricas ayudan en la comprensión de lo que puede ser cada uno de los métodos mencionados arriba, pero para entender mejor es necesario tratar de asociar tales métodos a su utilización en aplicaciones concretas.

En términos generales las siguientes son algunas de las posibilidades de aplicación de las técnicas bibliométricas, cienciométricas e informétricas:

* identificar las tendencias y el crecimiento del conocimiento en un área;

* identificar las revistas del núcleo de una disciplina;

* medir la cobertura de las revistas secundarias;

* identificar a los usuarios de una disciplina;

* prever las tendencias de publicación;

* estudiar la dispersión y la obsolescencia de la literatura científica; 
prever la productividad de autores individuales, organizaciones y países;

- medir el grado y los patrones de colaboración entre autores;

analizar los procesos de cita y co-cita;

determinar el desempeño de los sistemas de recuperación de información;

evaluar los aspectos estadísticos del lenguaje, las palabras y las frases;

evaluar la circulación y el uso de documentos en un centro de documentación;

medir el crecimiento de determinadas áreas y el surgimiento de nuevos temas.

El uso de técnicas bibliométricas puede contribuir de forma decisiva en épocas de recursos escasos, cuando un bibliotecario debe resolver qué títulos o publicaciones periódicas pueden, o no, ser suprimidos de una biblioteca. Se pueden obtener así indicadores de uso para definir una lista de publicaciones periódicas prioritarias y para prever la demanda futura. Es fundamental poder detectar la utilización real de los títulos existentes en una biblioteca y poder determinar la obsolescencia de las colecciones (Rivas, 1984). Los indicadores bibliométricos también son utilizados para evaluar la productividad y la calidad de la tarea de investigación de los científicos mediante la medición basada en el número de publicaciones y de citas de los distintos investigadores (Meis $\notin a l ., 1999)$.

En ese mismo orden de ideas, también Braga (1974, p. 162) señala que:

Para generalizar estadísticas empíricas... la Bibliometría examina, ante todo, las relaciones entre diferentes variables: recursos humanos-documentos, artículos-periódicos, producción-consumo, etc., que presentan diversos patrones de distribuición. El número de artículos que originam ncitas, el número de instituiciones que producen anualmente ndoctorados, el número de revistas que contienen nartículos - constituyen ejemplos del mismo tipo de distribución.

En lo que respecta a los métodos cienciométricos, según Callon đal. (1993), éstos se aplican principalmente al tratamiento y a la gestión de las informaciones formales provenientes de bases de datos científicas o técnicas. Incluso, según estos autores, las empresas utilizarían las técnicas cienciométricas para conseguir determinar cuál es la estrategia tecnológica seguida por sus competidores. Las empresas pueden, de esta manera, analizar las patentes, por ejemplo, identificando los principales temas de investigación y los actores significativos en un campo científico de su interés. Pueden, asimismo, detectar las especialidades científicas que sirven de base a sus tecnologías-clave. Por otra parte, documentos tales como las tesis también comienzan a ser objeto de análisis cienciométricos por parte de las empresas, con el objetivo específico de determinar qué asuntos, temáticas y caminos están siendo explorados en un determinado momento en el ambiente académico, lo que se constituye en una buena fuente de información para aquéllos que desean detectar los desarrollos más recientes. 
Es interesante destacar, por lo tanto, la creciente interacción entre ciencia y tecnología como uno de los espacios más fecundos para la investigación cienciométrica. El relevamiento de la interface entre ciencia y tecnología, como apunta Van Raan (1997), se convierte en uno de los ámbitos más ricos para el desarrollo y la utilización de los métodos cienciométricos. Las técnicas relativas a los análisis de co-cita y co-aparición de palabras, por ejemplo, son usadas para trazar un perfil de los campos científicos (a partir de datos sobre publicación) y tecnológicos (a partir de datos sobre patentes), posibilitando una cartografía de la ciencia y de la tecnología que incluya las fronteras de cada disciplina, la posición de los principales actores dentro del mapa y las representaciones específicas de cada una de las ramas del conocimiento.

Los indicadores infométricos, a su vez, ofrecen nuevas posibilidades para quien desee, por ejemplo, utilizar las bases de datos como instrumento de análisis. Pueden ser aplicados para evaluar los logros de las investigaciones, analizando no solamente la recuperación de la información, sino también los resultados de las búsquedas y su combinación con otras informaciones, a fin de mejorar la propia recuperación y la eficiencia en el acceso a la información y aun para economizar tiempo en el proceso de búsqueda. Conociendo las propiedades cuantitativas de la información contenida en los sistemas, la distribución de los términos usados en las búsquedas y la frecuencia de la aparición de los términos en una base, se pueden establecer correlaciones probabilísticas entre frecuencia de uso y de aparición de los términos que permitan mejorar sensiblemente el desempeño del sistema de recuperación. El sistema podrá, así, seguir el modelo de recuperación de información que más se adapte a las necesidades del usuario contemplando, también, las posibilidades de espacio de los equipos, los recursos con que cuente la unidad de información y las facilidades de actualización que cada modelo ofrezca (Wormell, 1998; Wolfram, 1992a; Wolfram, 1992b).

Cabe asimismo destacar que los análisis cuantitativos, con los avances tecnológicos, se ven facilitados y al mismo tiempo encuentran nuevos y estimulantes campos de actuación. Es el caso de los estudios que se comenzaron a desarrollar recientemente sobre el contenido y la estructura de las homepagesen la Wäb Según Almind \& Ingwersen (1997), estaría naciendo una nueva área de interés dentro de la informetría: la Webomerics Este subcampo apuntaría al estudio y a la cuantificación de la presencia de los diversos países en la red, de las proporciones de páginas personales, comerciales e institucionales, y al análisis de los hypedinks (que correspondería al tradicional análisis de citas en publicaciones impresas).

McGrath (cit. por Macias-Chapula, 1998) presenta un cuadro comparativo para la definición y clasificación de la bibliometría, cienciometría e informetría. Agregándole a esta tabla otras informaciones complementarias, podremos entender mejor las aplicaciones de estos tres subcampos. 
Cuadro1 - Aplicacianes delos distintos mítobs arantitativos

\begin{tabular}{|c|c|c|c|}
\hline Tipología & Bibliometría & Cienciometría & Informetría \\
\hline $\begin{array}{l}\text { Objeto de } \\
\text { estudio }\end{array}$ & $\begin{array}{l}\text { Libros, documentos, } \\
\text { revistas, artículos, auto- } \\
\text { res, usuarios }\end{array}$ & $\begin{array}{l}\text { Disciplinas, temas, áreas y } \\
\text { campos científicos y tec- } \\
\text { nológicos. } \\
\text { Patentes, disertaciones y } \\
\text { tesis }\end{array}$ & $\begin{array}{l}\text { Palabras, documentos, ba- } \\
\text { ses de datos, comunicacio- } \\
\text { nes informales (incluso en } \\
\text { ámbitos no científicos), ho } \\
\text { mepagesen la WWW }\end{array}$ \\
\hline Variables & $\begin{array}{l}\text { Número de préstamos } \\
\text { (circulación) y de citas, } \\
\text { frecuencia de la exten- } \\
\text { sión de frases }\end{array}$ & $\begin{array}{l}\text { Factores que diferencian } \\
\text { las subdisciplinas. } \\
\text { Modo en que se comuni- } \\
\text { can los científicos }\end{array}$ & $\begin{array}{l}\text { Difiere de la cienciometria } \\
\text { en el propósito de las va- } \\
\text { riables, p. ej., medir la re- } \\
\text { cuperación, la relevancia, } \\
\text { la revocación }\end{array}$ \\
\hline Métodos & $\begin{array}{l}\text { Ranking, frecuencia, } \\
\text { distribución }\end{array}$ & $\begin{array}{l}\text { Análisis de conjunto y de } \\
\text { correspondencia, co-apari- } \\
\text { ción de términos, expre- } \\
\text { siones, palabras-clave, } \\
\text { etcétera. }\end{array}$ & $\begin{array}{l}\text { Modelo vector-espacio, } \\
\text { modelos booleanos de re- } \\
\text { cuperación, modelos pro- } \\
\text { babilísticos; lenguaje de } \\
\text { procesamiento, abordajes } \\
\text { basados en el conocimien- } \\
\text { to, tesauros }\end{array}$ \\
\hline Objetivos & $\begin{array}{l}\text { Asignar recursos: per- } \\
\text { sonal, tiempo, dinero, } \\
\text { etcétera. }\end{array}$ & $\begin{array}{l}\text { Identificar campos de inte- } \\
\text { rés. Lugares de concentra- } \\
\text { ción temática. } \\
\text { Comprender cómo y cuán- } \\
\text { to se comunican los cientí- } \\
\text { ficos }\end{array}$ & $\begin{array}{l}\text { Mejorar la eficiencia de la } \\
\text { recuperación de la infor- } \\
\text { mación, identificar estruc- } \\
\text { turas y relaciones dentro } \\
\text { de los diversos sistemas de } \\
\text { información }\end{array}$ \\
\hline
\end{tabular}

Fuente: Adaptado de McGrath (cit. por Macias-Chapula, 1998).

Las definiciones apuntadas y el cuadro comparativo de los conceptos de bibliometría, cienciometría e informetría pueden ser representadas de manera que se pueda visualizar mejor la interrelación entre estos subcampos dentro de la Ciencia de la información, con el siguiente diagrama:

\section{A - Bibliometría \\ B - Cienciometría \\ C - Informetría}

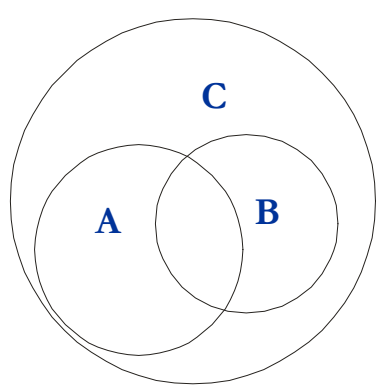


Esta representación está de acuerdo con los análisis de Gorkova (cit. por Brookes 1990), Russell (1994) y Tague-Sutckiffe (1992), quienes consideran que la informetría es más amplia y engloba a la bibliometría y la cienciometría. En las palabras del último autor, "theteminfomerics has beemecommon nlyin thepastiveyears, as general fiedofsudy whichindudestheendierfieds ofbibliamericsandieniomenics". ${ }^{3}$ La representación gráfica refleja, también, la superposición que en algún momento se da entre la bibliometría y la cienciometría, en la medida en que ésta aplica técnicas bibliométricas para la medición de la ciencia, mientras que la bibliometría, por su parte, es una rama del conocimiento con alcance multidisciplinario.

\section{CONCLUSIONES}

En definitiva hay algunos factores que diferencian un subcampo de otro, según sus definiciones teóricas, sus objetivos específicos, sus objetos de estudio y, principalmente, sus aplicaciones. Cuando no se tienen claramente definidos los conceptos de cada subcampo, sus variables y sus posibles aplicaciones, estas tres técnicas pueden ser confundidas y tomadas una por la otra, como ya sucedió y aún sucede en la literatura. Como afirma Russell (1994), el consenso alcanzado en algunos encuentros de especialistas indicando que la informetría era el campo que agrupaba o incluía a las otras técnicas (bibliometría y cienciometría) estaría nuevamente en discusión. Esta autora señala que artículos como los de Glänzel \& Schoepflin (1994), que buscan reinstalar la supremacía de la bibliometría, no ayudarán mucho a resolver la confusión teórica y el caos terminológico en lo que se refiere al ámbito, a las acciones y a las interrelaciones entre las tres especialidades. Tampoco ayudarían aquellos autores que, tal como Krauskopf (1994), sugieren nuevos términos para substituir los ya existentes (epistemometría en lugar de cienciometría), a pesar de la excelente argumentación y racionalidad de sus análisis.

Sería fundamental que fueran bien aclarados los metódos, funciones, y formas de utilización de acuerdo con los conceptos, alcance, objeto de estudio, calificación y características propias de cada uno de los tres subcampos. Como sostiene Rousseau (1994), debemos consolidar la idea de que todas estas especialidades encuentran su vínculo a partir de un origen común: todas ellas indudablemente constituyen subcampos de la Ciencia de la Información. Considerándolas de esta manera podemos comprender más satisfactoriamente la aplicabilidad de cada una y la utilidad que ofrecen a las más diversas áreas del conocimiento.

3 El término informetría se ha vuelto común sólo durante los últimos cinco años como un campo general de estudio que incluye a los anteriores campos de bibliometría y cienciometría. 


\section{REFERENCIAS BIBLIOGRÁFICAS}

Almind, Tomas C.; Ingwersen, Peter. Informetric analyses on the world wide web: methodological approaches to 'Webometrics'. Jaumal of Doamentation v. 53, n. 4, p. 404-426, 1997.

ALves, Rubem. Professor não vale nada. Fdha deSãoPaulo São Paulo, 12 set. 1999. Caderno Especial: Ranking da Ciência, p. 7.

BRAGA, Gilda Maria. Informação, ciência, política científica: o pensamento de Derek de Solla Price. Ciênia da Informaçãa v.3, n.2, p. 155-177, 1974.

BROOKES, B.C. Biblio-, sciento-, infor-metrics??? What are we talking about? En: Egghe, L.; Rousseau, R. (Eds.) Infometrics 89/ 90. Amsterdam: Elsevier, 1990. p.31-43.

CAllon, Michel; Courtial, Jean-Pierre; Penan, Hervé. Ciencome túa la medición de la actividad científica: de la bibliometría a la vigilancia tecnológica. Gijón: Trea, 1995. 110 p.

Castro, Cláudio de Moura. Ciênda eunivessidade Rio de Janeiro: Zahar, 1985. 56 p.

Dobrov, G.M.; Karennoi, A.A. The informational basis of scientometrics. En: Mikhailov, A.I. et. al. (Eds.) Ontheretical prddems of infomatics Moscou: VINITI. FID, 1969. p.165-191.

EGGHE, L. Bridging the gaps: conceptual discussions on informetrics. Saientamerics Amsterdam, v.30, n. 1, p.35-47, 1994.

FONSECA, Edson Nery da. Bibliografia estatística e bibliometria: uma reivindicação de prioridades. Ciência da Informaçãa Rio de Janeiro, v. 2, n.1, p. 5-7, 1973.

--- (Org.). Bibliamenia teoria e prática. São Paulo: EDUSP, 1986. $141 \mathrm{p}$.

GLÄNZEL, W.; Schoepflin, U. Little scientometrics, big scientometrics... and beyond? Saientameris Amsterdam, v.30, n. 2-3, p. 375-384, 1994.

HECKLER, Evaldo; Back, Sebald; Massing, Egon Ricardo. Didio nério mondógico da línga partuguesa. São Leopoldo, RS: UNISINOS, 1984. v. 4.

IRVINE, J.; MARTIN, B. R. International comparisons of scientific performance revisited. Säentantrics Amsterdam, v. 15, n. 5-6, p. 369-392, 1989. 
INSTITUTE FOR SCIENCE INFORMATION (ISI). Produdsandserv[online] Disponible en Internet vía WWW. URL: http://www.isinet.com/isi/products. Archivo capturado el $12 / 10 / 00$.

KRAUSKOPF, M. Epistemometria, a term contributing to express the meaning and potential methodologies of scientometrics in spanish speaking countries. Saientomenis, v. 30, n. 2-3, p. 424428, 1994.

LAWANI, S. M. Bibliometrics: its theoretical foundations, methods and applications. Libri, v. 31, n. 4, p. 294-315, 1981.

MACIAS-CHAPULA, César A. O papel da informetria e da cienciometria e sua perspectiva nacional e internacional. Ciênia daInfomaçãa Brasília, v. 27, n. 2, p. 134-140, maio/ago. 1998.

MEIS, Leopoldo de; Maia, Cristina; Lannes, Denise; Machado, Rita Pinheiro. Uso de indicadores exige cautela. Folha deSão Paulo São Paulo, 12 set. 1999. Caderno Especial: Ranking da Ciência, p. 7.

OliveIRA, Admar Costa de; Dórea, José Garrofe; Domene, Semíramis Martins Alvares. Bibliometria na avaliação da produção científica da área de nutrição registrada no Cibran: período de 1984-1989. CiêniadaInfomaçãa Brasília, v. 21, n. 3, p. 239-242, set./dez. 1992.

Pritchard, Alan. Statistical bibliography or bibliometrics? Joumal of Doumentation v. 25, n. 4, p. 348-349, dec. 1969.

RAVICHANDRA RAO, I.K. MéodosquantitativosemBiblideronomiae Ciênia da Infomaçãa Brasília: ABDF, 1986. 272 p.

RIVAS, Liliana M. Técnicas bibliométricas: selección y evaluación de publicaciones periódicas para bibliotecas y bases de datos biomédicas especializadas. Biblidtedoǵa y Doumentación Buenos Aires, v. 6, n. 6-11, p. 41-81, jul/dic. 1981 - ene./jun. 1984.

RousSEAU, Ronald. Similarities between Informetrics and Econometrics. Saientamtrics Amsterdam, v.30, n. 2-3, p.385-387, 1994.

RUSSELL, Jane M. Back to the future for informetrics? Saientome trics Amsterdam, v.30, n.1, p.407-410, 1994.

SANDSTROM , Alan R.; Sandstrom, Pamela Effrein. The use and misuse of anthropological methods in library and information science research.. Lybrary Quarterly, v. 65, n. 2, p. 161-199, apr. 1995. 
SENGUPTA, I. N. Bibliometrics, informetrics, scientometrics end librametrics: an overview. Libri, v. 42, n. 2, p. 99-135, 1992.

SHAPIRO, Fred R. Origins of bibliometrics, citation indexing, and citation analysis: the neglected legal literature. Jamal of the Ameican SocietyforInfomation Saience(JASIS), v. 43, n. 5, p. 337 339, 1992.

SPINAK, Ernesto. Dicionarioenadopédicodebibliomeńa, denaiamenáa einfomenáa. Montevideo, 1996. 245 p.

- - - Indicadores cienciométricos. Ciênaiada Informaçãa Brasília, v. 27, n. 2, p. 141-148, maio/ago. 1998.

TAGUE-SUTCKIFFE, Jean. An introduction to informetrics. Information Processing \& Managagement, Oxford, v. 28, n. 1, p. 1-3, 1992.

TORRINHA, Francisco. Diaiańniopartuguêslatina Porto: Ed. Domingos, 1939. 1129 p.

VAN RAAN, A.F.J. Scientometrics: state-of-art. Säentometics Amsterdam, v. 38, n. 1, p. 205-218, 1997.

VELHO, Léa Maria L.S. Como medir a ciência? RevistaBrasileirade Tendogja, Brasília, v. 16, n. 1, p.35-41, jan./ fev. 1985.

WOLFRAM, Dietmar. Applying informetric characteristics of database to ir system file design, part I: informetric models. Information Processing \& Managagment, Oxford, v. 28, n. 1, p. 121-133, 1992a.

WOLFRAM, Dietmar. Applying informetric characteristics of database to ir system file design, part II: simulation comparisons. Infomation Procssing \& Managagment, Oxford, v. 28, n. 1, p. 135-151, 1992b.

WORMELL, Irene. Informetria: explorando bases de dados como instrumentos de análise. CiêniadaInfomaçãa Brasília, v. 27, n. 2, 1998. [online] Disponible en Intenet vía WWW. URL: www.scielo.br/cgi-bin/fbpe/. Archivo capturado el 16/06/99.

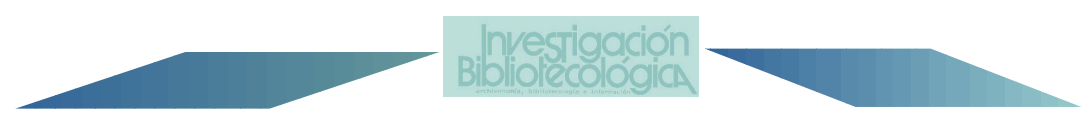

